\title{
ONLINE HANDWRITING SIGNATURE: AN EXPERIMENTAL STUDY ON PRESSURE SIGNAL NORMALIZATION IN DIFFERENT DEVICES FOR BIOMETRIC ACQUISITION
}

\author{
FABIO GARZIA ${ }^{1}$, MARIA CHIARA GIANNONE ${ }^{1} \&$ MARCOS FAUNDEZ-ZANUY $^{2}$ \\ ${ }^{1}$ Safety and Security Engineering Group - DICMA, SAPIENZA - University of Rome, Italy \\ ${ }^{2}$ Tecnocampus, Universitat Pompeu Fabra, Spain
}

\begin{abstract}
Online handwriting signature is an important element in biometrics and therefore for security applications. In this paper the characterization of pressure for different acquisition devices for digital signature is presented, considering that technological evolution always produces new devices and that is why it is necessary to facilitate interoperability between them. The devices used are three different Wacom tablets: Wacom Intuos pro, Wacom Cintiq 16 and STU-530. Each of them has different pens and more than one nib and that is why for each of them over one case was analyzed in reference to the different pens used. It was experimented how, thanks to the respective software of the tablets, it is possible to acquire the pressure that is generated by the pen on the tablet and to connect it to the weight force exerted by them thanks to a balance placed under the tablet. The process used allowed to create for each tablet the pressure/weight plot that permitted to derive the different sensitivities of the tablets to pressure and also the different saturation values. In addition, it was also analyzed the variation of the thickness of the stroke exerted by the pen going to write on the tablet with different values of force applied. Thanks to the use of carbon copy paper, which allowed to reproduce the line generated by the pen on a sheet of paper, it was then possible to study under the microscope the trend of the thickness of the line drawn as the force varies. The obtained results allowed to obtain a direct comparison between the signals acquired by different devices thanks to the normalization processes that permit to increase the recognition performance.

Keywords: online handwriting signature, pressure, software, thickness, normalization processes.
\end{abstract}

\section{INTRODUCTION}

Online handwritten analysis presents many applications in e-security, signature biometrics being the most popular but not the only one [1]. From a human behavior and health condition perspective, online handwriting biometrics are more appealing and informative than other popular biometrics traits such as fingerprints or iris [2], [3]. It can be noted that nowadays, most of the published research in biometric signal processing is based on image and speech. This might be linked to the fact that these signals are simpler and cheaper to acquire compared with others such as online handwriting where specific digitizing tablets are needed [1].

Fortunately, online handwriting signals are more present in our society than a few years ago due to the increasing popularity of tactile devices and their corresponding cost reduction [4]. As a result, we forecast a considerable growth in applications in this field. Indeed, in a near future, these pen-based handheld devices (tablets, phablets, cellphones, etc.) will help to protect people's sensitive data, for example, incorporating automatic signature verification and writer identification [1].

Digitizing tablets for handwritten tasks are general devices that can be easily found in almost any computer shop. They are mainly sold for graphic design. However, some small devices exist for biometric online signature acquisition, for instance in a post office or for VISA/Mastercard payment. This later case are very small tablets with lower quality than the most popular ones for graphic design. On the other hand, scientific community has been using 
all these tablets intensively for e-health and e-security analysis based on handwritten tasks. Calibrated tablets do not exist as they have not been developed for instrumentation. In one case, because only the graphic result and comfort for the user is important. In the other case, because it is just a substitute of the classic ink and paper method, and the goal is to save paper. The signature is not going to further treatment nor processing so accuracy of pressure is not really an issue. In fact, classic ink plus paper method does not consider pressure at all. Thus, rather than a gold standard or reference behaviour, a precision calibrated balance to obtain accurate measurements is used in the present work, as described in the following.

Regarding the safety of online handwritten, among the different signal functions, pressure is one of the most important because it has a discriminative power in e-security and e-health. In fact, in the medical field, the study of handwriting has proven to be useful in diagnosing and tracking some diseases of the nervous system [5].

In an online context, pressure can be measured directly from sensors embedded in the stylus, providing a continuous signal sampled at a given frequency and amplitude resolution. When the stylus is used with a tablet, $\mathrm{x}$ and $\mathrm{y}$ coordinates versus time are also available as well as tilt and azimuth angles. When the stylus is instrumented, other signals like acceleration can also be provided [6], even if previous works were made with limited resources also from precision of the used balance point of view (0.1 grams instead of 0.01 grams of the present work) [6]. Because of this, in this paper, the pressure signal normalization of three graphics tablets was analyzed. The chosen graphics tablets are from the Wacom company, which was founded in 1983. It is a graphic tablet and graphic design company based in Ôtone, Japan. Wacom's innovation lies in this active digitizer technology that supports up to 2056 levels of pressure, providing the Tablet PC with advanced digital ink capabilities and the ability to write naturally on the screen by resting your wrist on it. The three tablets chosen are the STU-530, Cintiq 16 and the Intuos Pro. Each has different features and different pens to use.

The study is focused on experimental tests that allow us to collect a set of pairs of values using the high precision balance FR-500, the Microscope OPTIKA XDS-3MET and the different acquisition software for each tablet. The values collected are the pressure level provided by the tablet and the weight measured in the balance. In addition, several tests have shown that as the pressure used to generate the line on the graphic tablet increases, the thickness of the line also changes, which allows us to understand how the mark is influenced by the force used to write on the tablet.

The study represents a significant improvement of previous works [1], [5], [6] as it is based on three different pens with seven different nibs instead of a single tablet and only two different pens (ink and plastic). Further, previous paper [6] had not deal a lot with ink trace measurement while the present work goes under very complete stroke analysis by means of an electronic microscope and these results are shown in the following. Moreover, a special wood device to measure the widths of both sides of the nib has been developed, while the previous paper [6] was limited to only one side (end).

The significance of this study is in e-health as some pathologies reveal a loss of pressure [7] and in e-security too, as some published papers [6] demonstrate the positive contribution of pressure information to biometric rates (identification and verification). In this paper the possibility to deal with signals acquired by different pens and tablets is opened. This is particularly important because current state of the art is mainly limited to situations where all the experiments are performed with same tablet and pen model, and this is could not represent a real situation [8]. 
The paper is organized as follows: Section 2 is devoted to the characterization of the three handwriting devices, Section 3 presents the methodology and data Sample analysis, Section 4 the experimental results, Section 5 the conclusions.

\section{CHARACTERIZATION OF HANDWRITING DEVICES}

For this study, three different Wacom digitizing tablets have been evaluated. Each model has several writing styluses (pens) to be tested, as shown in Table 1.

Table 1: Wacom tablets and pens analysed (lpi $=$ lines per inch).

\begin{tabular}{|l|c|c|c|}
\hline Characteristics tablet & STU-530 & Intuos Pro & Cintiq 16 \\
\hline Acquisition area & $108 \times 65 \mathrm{~mm}$ & $430 \times 287 \mathrm{~mm}$ & $422 \times 285 \mathrm{~mm}$ \\
\hline Pressure range & {$[0,1023]$} & {$[0,7557]$} & {$[0,32767]$} \\
\hline Coordinates precision & $\begin{array}{c} \pm 0.02 \mathrm{inches} / \\
\pm 0.5 \mathrm{~mm} \\
\text { (center) }\end{array}$ & - & - \\
\hline $\begin{array}{l}\text { Resolution of coordinates } \\
\text { (non-interpolated) }\end{array}$ & $\begin{array}{c}2540 \mathrm{lpi} \\
0.01 \mathrm{~mm} / \mathrm{pt}\end{array}$ & $5080 \mathrm{lpi}$ & $5080 \mathrm{lpi}$ \\
\hline $\begin{array}{l}\text { Report rate } \\
\text { (non-interpolated) }\end{array}$ & $\begin{array}{c}200 \text { points per } \\
\text { second }\end{array}$ & $\begin{array}{c}200 \text { points per } \\
\text { second }\end{array}$ & - \\
\hline Pens & Plastic1 & $\begin{array}{c}\text { Plastic2 } \\
\text { Inkpen }\end{array}$ & $\begin{array}{c}\text { Plastic3 } \\
\text { Pro pen 3D }\end{array}$ \\
\hline
\end{tabular}
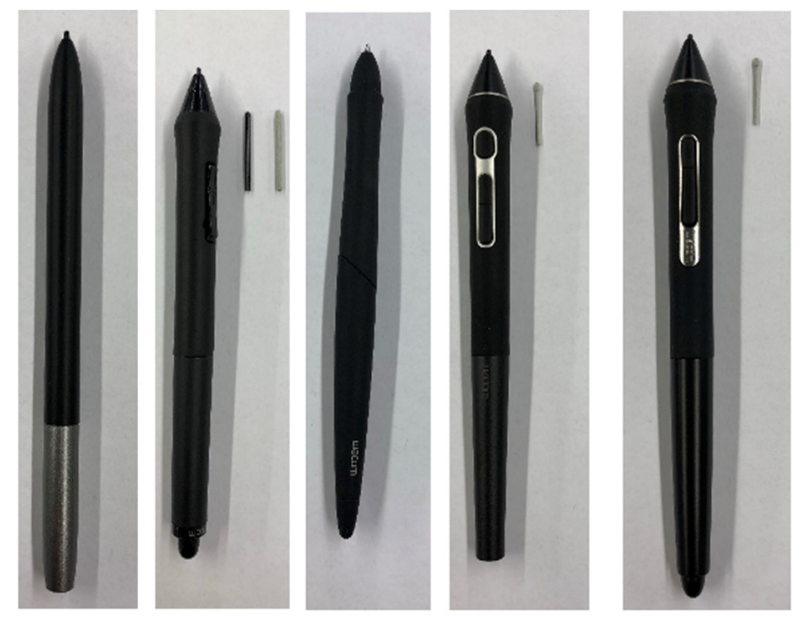

Figure 1: Pens and nibs used in the study.

\section{METHODOLOGY AND DATA SAMPLE ANALYSIS}

In this study, two different measurements were made for the respective graphic tablets. The first was made with the use of a FR-500 balance, which made it possible to measure the weight force exerted by the pen writing on the tablet and at the same time to capture, with the use of a software, the pressure exerted. The second was based on measuring the thickness of the line drawn by the pen thanks to the use of OPTIKA XDS-3MET Microscope and copying paper. 


\subsection{Experimental setup for pressure measurement}

The first measurement is made by placing the tablet on the scale, to capture the weight of each measurement, as shown in Fig. 2, and in the meantime, we use the software to see the pressure generated on the computer. Thanks to this, it is possible to create a plot weight/Tablet Value that allows to see the trend of the curve and the saturation point of the pressure [9]-[11]. The test was done for each graphic tablet with the different pens described above.

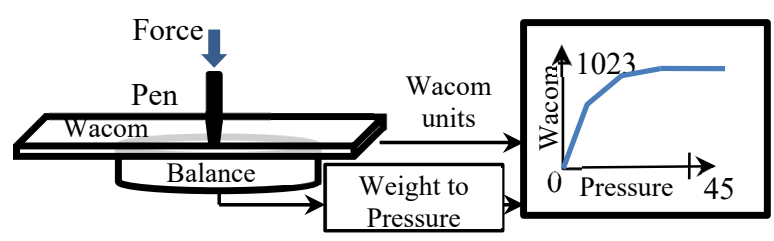

(a)

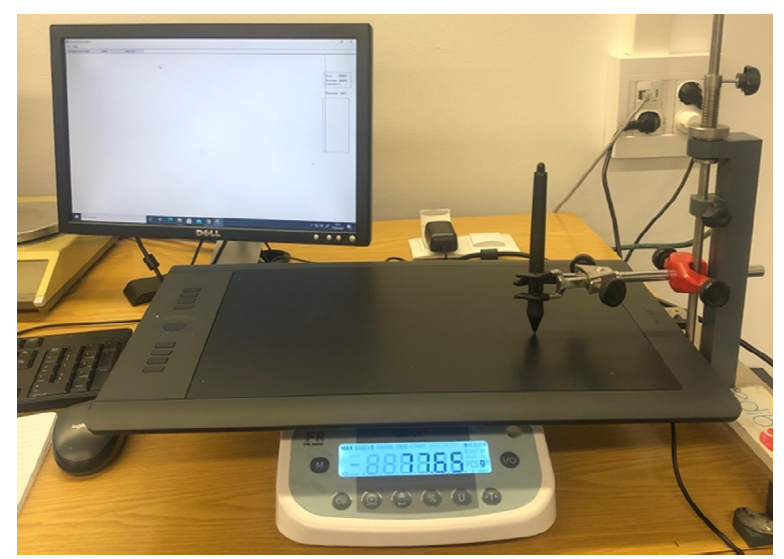

(b)

Figure 2: Configuration used for the analysis. (a) Schema for the experimental measurements to characterize the pen from [6]; and (b) Experimental setup (precision balance FR-500, stylus, and force actuator).

\subsection{Line thickness measurement setup}

The second study concerns the characterisation of pen nibs to determine the width of the strokes produced by different pens and tablets. A set of straights lines has been performed by hand trying to keep constant pressure during the whole realization. Then has been measured the width of each line with the help of the microscope OPTIKA XDS-3MET (https://www.optikamicroscopes.com/optikamicroscopes/product/im-3-series/) to evaluate and photograph the strokes. 
To measure with the help of the microscope the width of the lines, were produced lines on white sheets of paper show in Fig. 3. In the case of the plastic pens, we have used a carbon copy paper on the top of the white paper. Also, the widths for the ink pen with and without carbon copy paper were measured, although it is not necessary to measure the width, as it is able to produce lines on paper directly. This permits to validate the process used with the plastic pen and carbon copy. So, in Fig. 4 we can see the image captured by the microscope to measure the thickness.

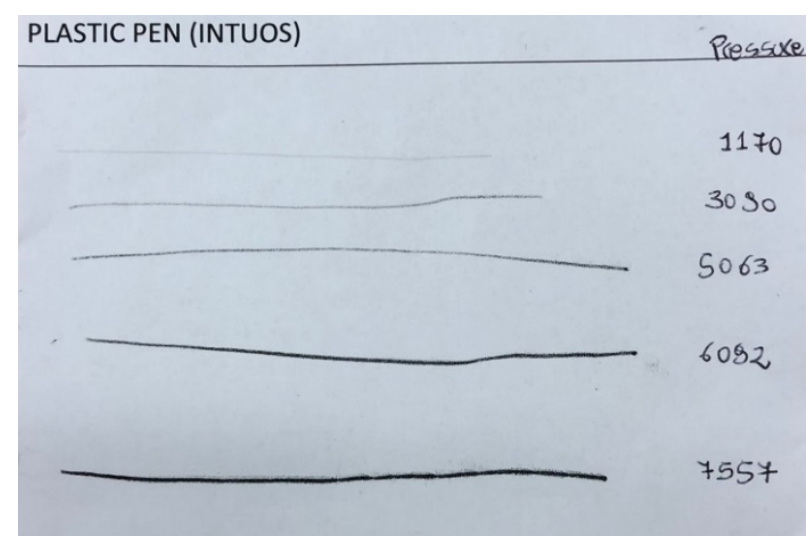

Figure 3: Example of the sheets (which are $13 \times 9 \mathrm{~cm}$ ).

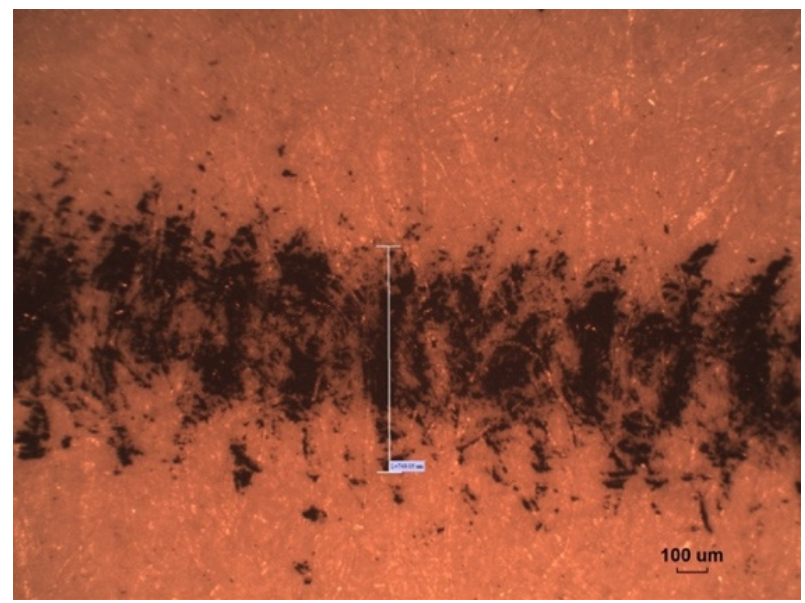

Figure 4: Straight line from the electronic microscope for the Intuos plastic pen.

\section{EXPERIMENTAL RESULTS}

The results obtained from these two studies have been plotted. For the first one, the tests carried out led to the construction of these graphs, while as far as the Intuos Pro plot is concerned, a study already carried out previously was taken [6].

So, comparing the weight obtained from the balance and the value obtained in the Wacom tablet, we find the experimental characteristic of the pressure sensor shown in Figs 5-7. 


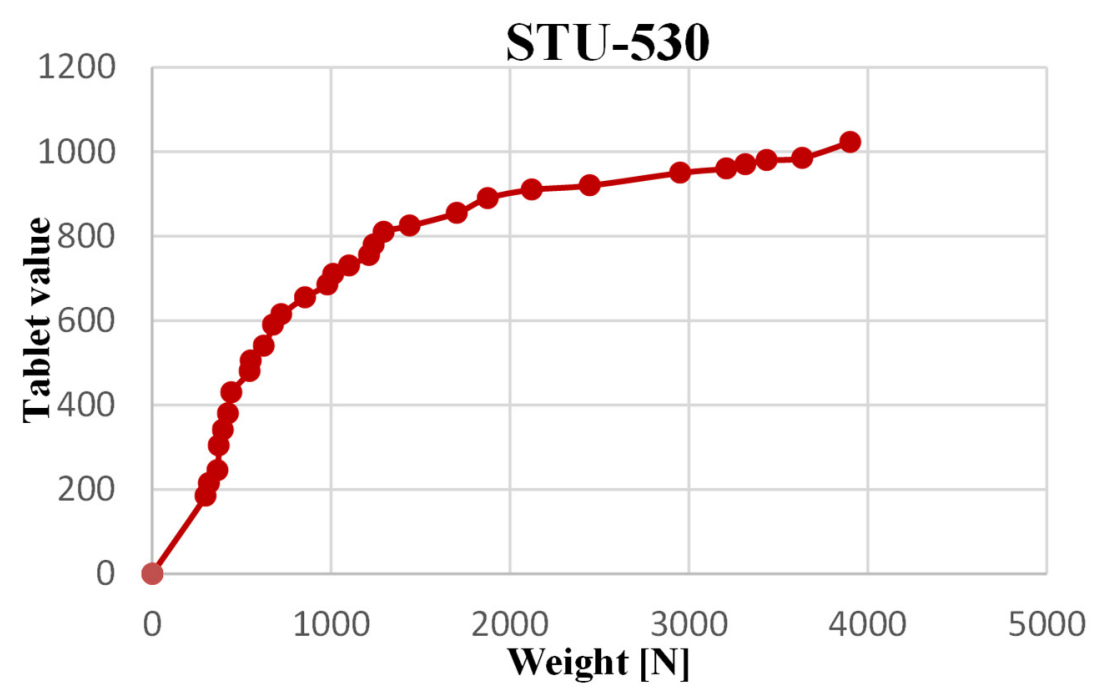

Figure 5: STU-530 Wacom tablet value obtained for different weight values.

\section{CINTIQ 16}

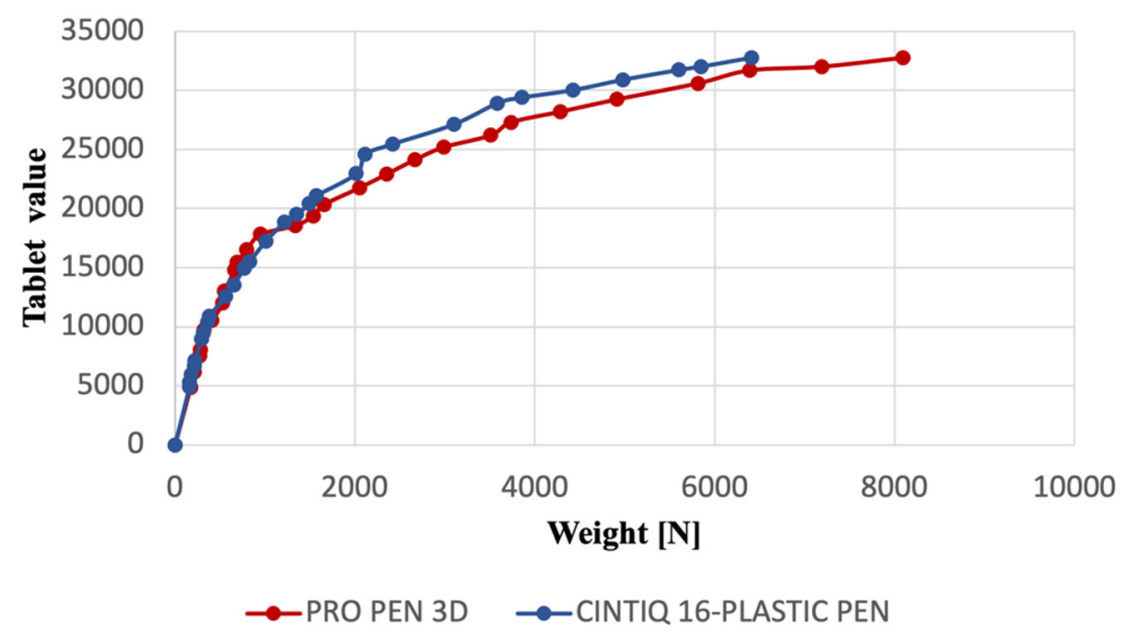

Figure 6: Cintiq 16 Wacom tablet value obtained for different weight values.

We observe in Fig. 7 that the plastic pen reaches saturation before the ink pen. Alternatively, the plastic pen has smaller dynamic range than the ink pen. This is not surprising since the ink pen has higher price than the plastic one, it must be purchased separately, and can be considered of better quality. However, its curve also exhibits a nonlinear behavior, which affects the obtained pressure values [6].

As for the microscopic study of the thickness of the line made with the different pens, it shows that in these graphs as the pressure increases, the thickness also increases. 


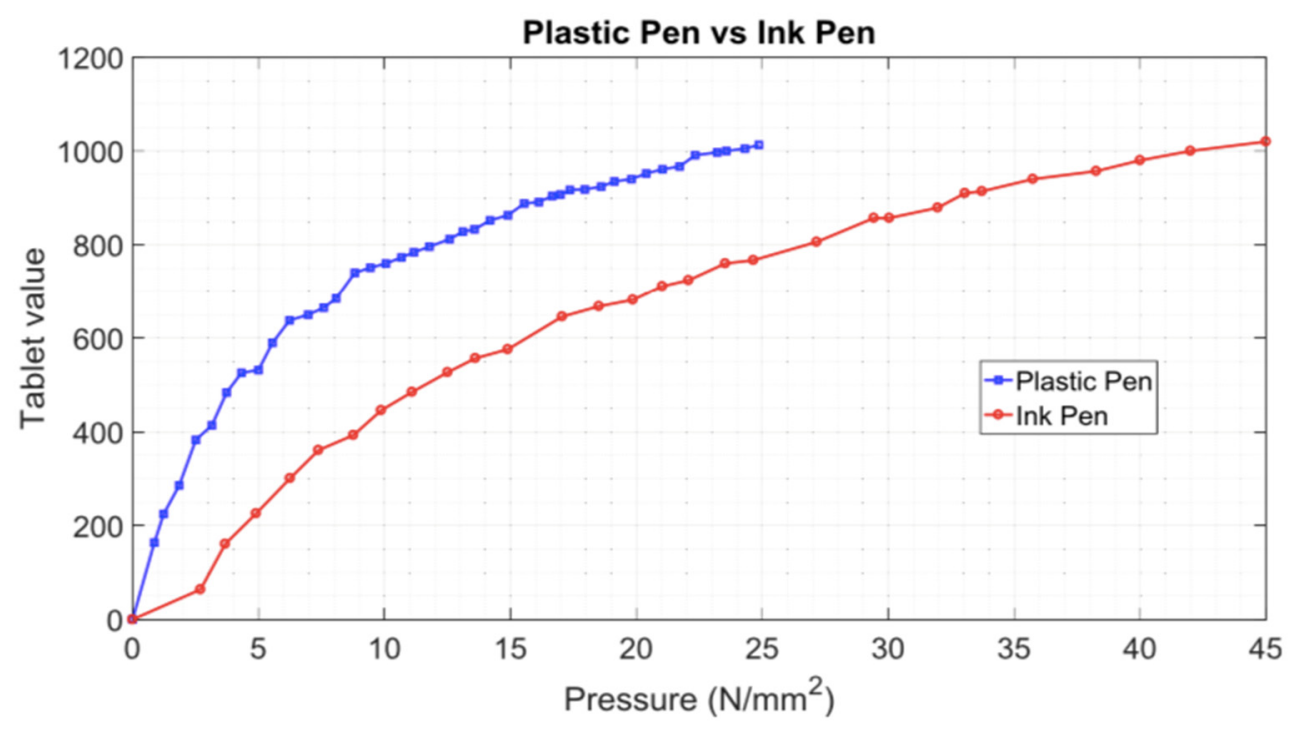

Figure 7: Intuos Wacom tablet value obtained for different pressure values [6].

Figs $8-10$ show the measurements for different pens and exerted pressures. Two important observations can be remarked:

- It shows that the measurements for the case of the ink pen are very similar with and without carbon copy paper. This means that the use of carbon copy paper is not distorting the width measurements and validates the experimental procedure for the plastic pens.

- The ink pen shows better permanence on the line width for different pressure levels, which remains more constant than for the plastic pens.

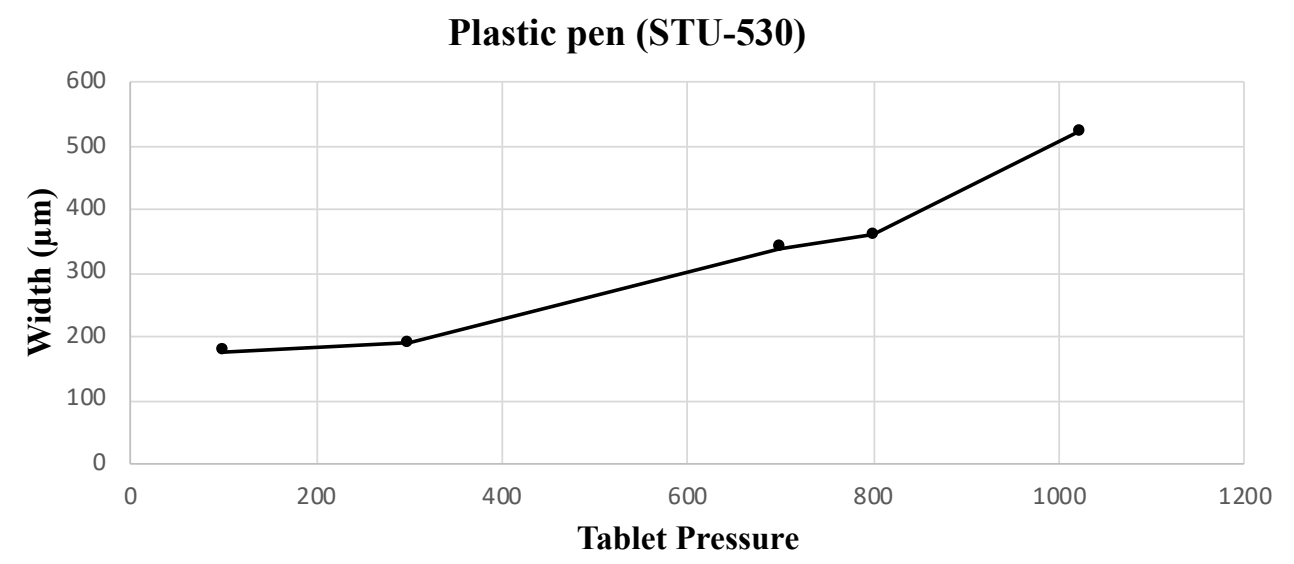

Figure 8: Width measurements for straight lines performed at different exerted pressures for the Wacom STU-530. 


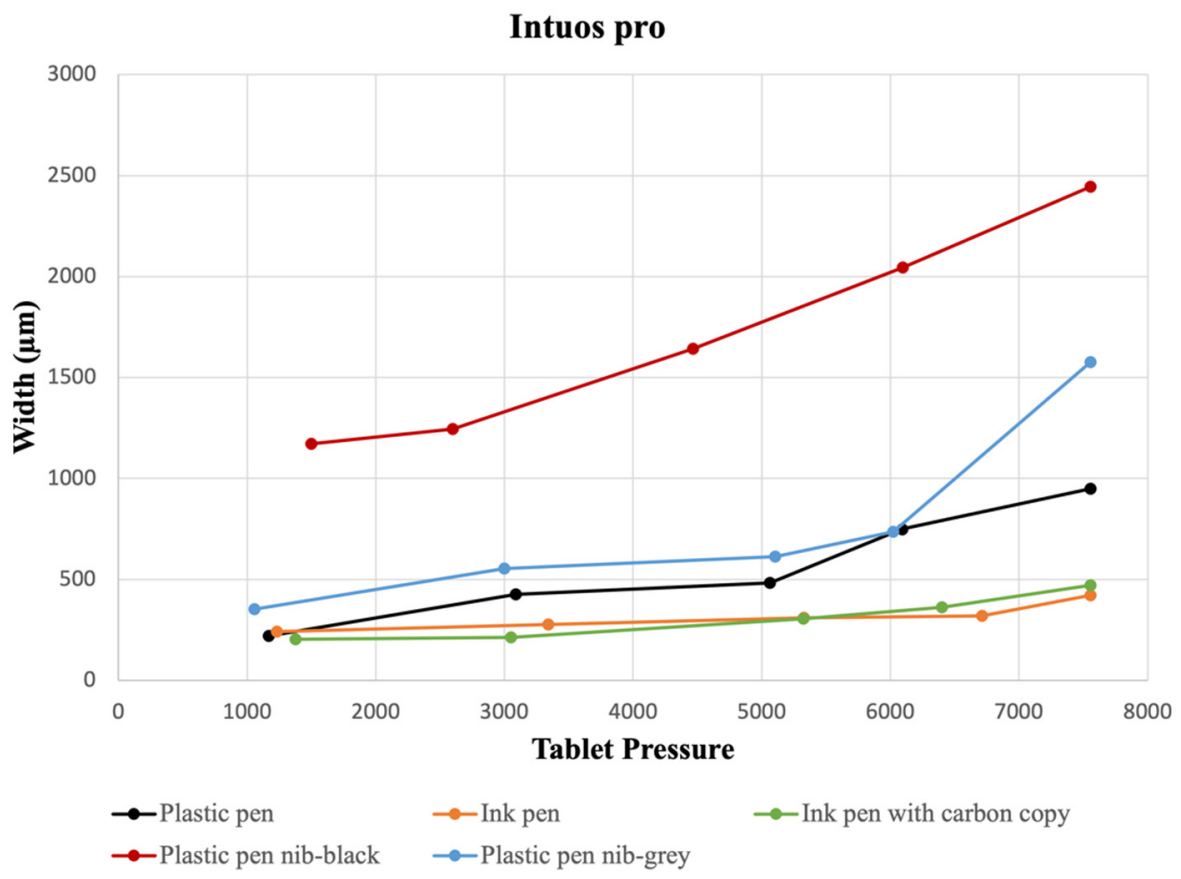

Figure 9: Width measurements for straight lines performed at different exerted pressures for Wacom Intuos Pro.

\section{Cintiq 16}

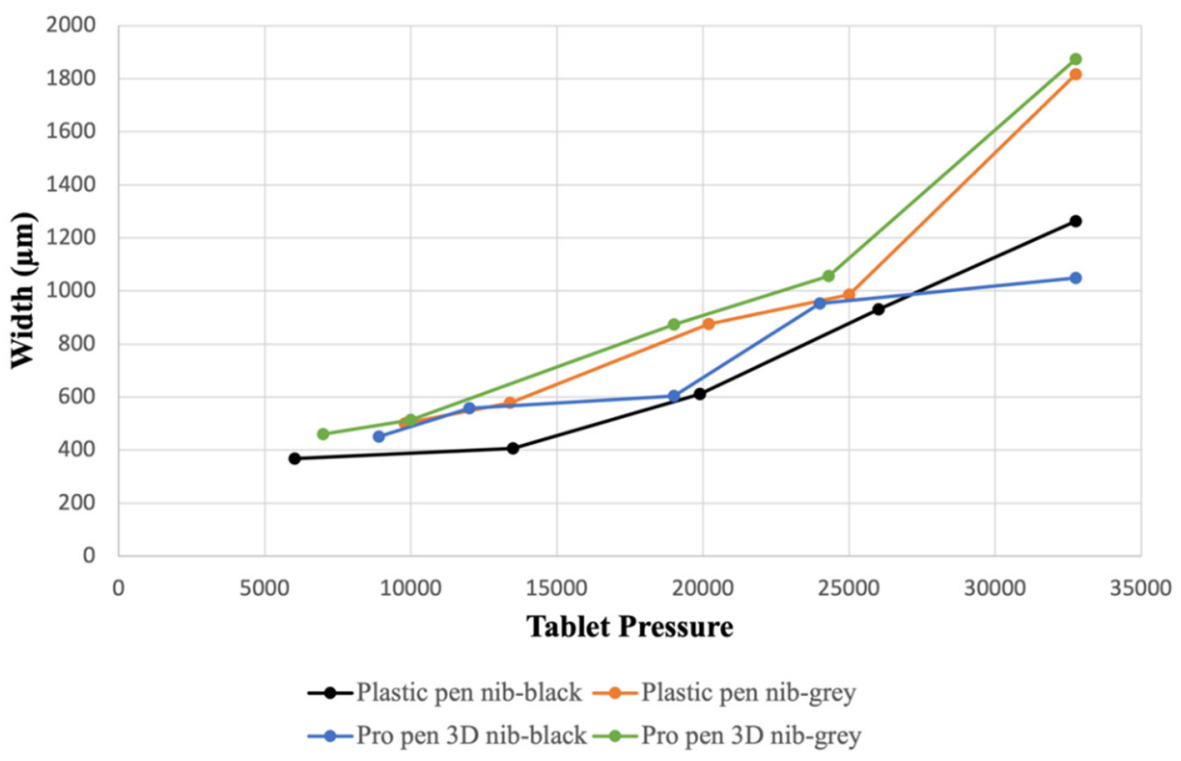

Figure 10: Width measurements for straight lines performed at different exerted pressures for Cintiq 16. 


\section{CONCLUSIONS}

In this paper, we have presented an experimental setup for pressure and line thickness measurement for the three different Wacom graphics tablets. The main conclusions are:

- The curve that emerges from the studies of the pressure exerted by the stylus generates an exponential curve with important saturation values for high pressures.

- The Intuos Wacom plastic pen reaches saturation for lower pressure than the Wacom ink pen, which presents a higher dynamic range (almost two times higher) and thus, it is has a better pressure sensor [6].

- The Cintiq Wacom plastic pen reaches saturation for lower pressure that the Wacom pro pen 3D.

- The study of the thickness of the lines made with the different stylus shows that the increase of pressure also affects the thickness, which grows. The stylus which shows the best performance is the ink pen which remains almost constant.

\section{ACKNOWLEDGEMENT}

This work has been funded by Spanish grant Ministerio de ciencia e innovación PID2020113242RB-I00.

\section{REFERENCES}

[1] Faundez-Zanuy, M., Fierrez, J., Ferrer, M.A. \& Plamondon, R., Handwriting biometrics: Applications and future trends in e-security and e-health. Cogn. Comput., 12, pp. 940-953, 2020. DOI: 10.1007/s12559-020-09755-z.

[2] De Stefano, C., Fontanella, F., Impedovo, D., Pirlo, G. \& Scotto di Freca, A., Handwriting analysis to support neurodegenerative diseases diagnosis: A review. Pattern Recogn. Lett., 121, pp. 37-45, 2019.

[3] Martinez-Diaz, M., Fierrez, J. \& Hangai, S., Signature matching. Encyclopedia of Biometrics, 2nd ed., eds S.Z. Li \& A.K. Jain, Springer, pp. 1382-1387, 2015.

[4] Tolosana, R., Vera-Rodriguez, R., Fierrez, J., Morales, A. \& Ortega-Garcia, J., Benchmarking desktop and mobile handwriting across COTS devices: The e-BioSign biometric database. PLoS One, 12(5), e0176792, 2017.

[5] Faundez-Zanuy, M. et al., A preliminary study of online drawings and dementia diagnose. Neural Nets and Surroundings: Smart Innovation, Systems and Technologies, eds B. Apolloni, S. Bassis, A. Esposito \& F. Morabito, vol. 19, Springer, Berlin, Heidelberg. DOI: 10.1007/978-3-642-35467-0_36.

[6] Faundez-Zanuy, M., Brotons-Rufes, O., Paul-Recarens, C. \& Plamondon, R., On handwriting pressure normalization for interoperability of different acquisition stylus. IEEE Access, 9, pp. 18443-18453, 2021. DOI: 10.1109/ACCESS.2021.3053499.

[7] Faundez-Zanuy, M. et al., A preliminary study of online drawings and dementia diagnose. Neural Nets and Surroundings: Smart Innovation, Systems and Technologies, eds B. Apolloni, S. Bassis, A. Esposito \& F. Morabito, vol. 19, Springer, Berlin, Heidelberg. DOI: 10.1007/978-3-642-35467-0_36.

[8] Faundez-Zanuy, M., On-line signature recognition based on VQ-DTW. Pattern Recognit., 40(3), pp. 981-992, 2007. DOI: 10.1016/j.patcog.2006.06.007.

[9] Schomakerand, L.R.B. \& Plamondon, R., The relation between pen force and penpoint kinematics in handwriting. Biol. Cybern., 63(4), pp. 277-289, 1990.

DOI: $10.1007 / \mathrm{BF} 00203451$. 
184 Safety and Security Engineering IX

[10] Alonso-Fernandez, F., Fierrez-Aguilar, J. \& Ortega-Garcia, J., Sensor interoperability and fusion in signature verification: A case study using tablet PC. Advances in Biometric Person Authentication (Lecture Notes in Computer Science), vol. 3781, eds S.Z. Li, Z. Sun, T. Tan, S. Pankanti, G. Chollet \& D. Zhang, Springer: Berlin, 2005, pp. 180-187. DOI: 10.1007/11569947_23.

[11] Antal, M. \& Bandi, A., Finger or stylus: Their impact on the performance of on-line signature verification systems. Proc. 5th Int. Conf. Recent Achievements Mechatron. Autom. Comput. Sci. Robot., pp. 11-22, 2017. 